\title{
Some studies on early embryonic development relevant to the study of cancer
}

\author{
H. R. WOODLAND \\ From the Medical Research Council Laboratory of Molecular Biology, Cambridge
}

Studies on early embryonic development are commonly directed at a causal analysis of cell differentiation. For the purpose of such experiments the word differentiation is commonly used to describe the acquisition of specialized functions by cells and it is therefore doubtful if these studies are directly relevant to an understanding of cancer. This is because cancerous cells vary from those which are very highly specialized, like those of a melanoma, to those which are unspecialized, but which form daughters with a wide variety of specialized functions like those of a teratoma. It is thus difficult to recognize any common feature of normal and malignant cells which relates both to their cancerous and to their differentiated states. The most obvious common feature of cancerous cells is that they divide rapidly and in a manner that appears to have escaped the normal regulatory mechanisms of the body. The normal development of an organism depends on the precise operation of such control mechanisms, and for this reason the embryo provides a potentially interesting situation in which to study the regulation of cell multiplication under normal conditions.

Two main types of embryological study which may help the student of cancerous cells are outlined in this brief review. The aim of the first is to understand the way in which the cell cycle is controlled in normal cells. The embryo provides a potentially rewarding situation for such studies because the frequency of cell division changes so greatly in early development. In the second type, the technique of nuclear transplantation is used. This procedure enables the experimenter to test the ability of a somatic cell nucleus to programme the development of a normal embryo. The development of a multicellular organism depends on the accurate control of cell division, and the ability of the nucleus to direct the organization of a wide variety of cells with different specialized functions. The assay of the developmental potentiality of the cancer cell nucleus by this technique would therefore help to show whether the appearance of the cancerous state depends on an irreversible change in the genetic material of the cell. This would be made manifest as an incapacity to direct normal cell specialization, or an inability to respond to the normal cytoplasmic regulation of nuclear reproduction.

\section{The Regulation of the Cell Cycle in Early Embryos}

Development may conveniently be said to begin with the growth of the oocyte and sperm in the parental gonad. Of these two cells the oocyte is of most fundamental importance in early development, for it provides the overwhelming part of the cytoplasm of the zygote. Usually the initial role of the sperm is to act as a stimulus for early development to begin. In many animals this stimulus is not necessary, and early development is nearly normal when the sperm makes no genetic contribution.

In early amphibian development the cell division cycle changes according to the following programme. The vitellogenic oocyte is a non-dividing, tetraploid, meiotic cell which grows for three months or more in the ovary, with intense RNA synthesis but no DNA synthesis (Izawa, Allfrey, and Mirsky, 1963; Gurdon, 1967). Hormonal changes trigger the nuclear and cytoplasmic divisions which complete meiosis, and the ovulation of the oocyte from the follicle. When the egg is fertilized the nuclei of the zygote replicate their DNA and they fuse during the division that ensues. This first cell cycle takes about 90 minutes. The daughter cells then divide rapidly, with cell cycles as short as 15 minutes and an Sphase of about 10 minutes (Graham and Morgan, 1966) until, as the end of the blastula stage of development approaches, the rate of cell division slows in a well defined way. This is characteristically different in the various regions of the embryo. The early gastrula produced by this phase of development is composed of about 30000 cells, which have taken 10 hours to be formed (Deuchar, 1958; Woodland and Gurdon, 1968).

The initial stages of early development thus involve great changes in the cell cycle. The magni- 
tude of these changes presents certain advantages in studying the control of cell division and DNA synthesis, particularly when they are compared with the relatively minor alterations of the cell cycle usually occurring in adult somatic cells. The oocytes and the eggs of Amphibia have another advantage, their large size. This makes it possible to inject substances into them without necessarily disturbing their normal behaviour, and thus to probe the regulatory mechanisms of the living cell. Experiments of this type have been outlined in more detail elsewhere (Gurdon and Woodland, 1968, 1970; Smith and Ecker, 1970; Woodland, Ford, Gurdon, and Lane, 1972). The injection of nuclei, DNA, and cytoplasm into eggs and oocytes has enabled the following conclusions to be drawn about these control mechanisms:

1 The cytoplasm of eggs has the property of inducing DNA synthesis in any nuclei it surrounds. This effect is the same whether the cells originally containing the nuclei normally synthesize DNA or not (Graham, Arms, and Gurdon, 1966).

2 Oocyte cytoplasm does not induce nuclear DNA synthesis. When the oocyte nuclear membrane breaks down during the hormonal conversion of an oocyte to an egg, its cytoplasm acquires the ability to induce synthesis (Gurdon, 1967).

3 When single somatic nuclei are transferred into an enucleated egg, normal development frequently ensues (Gurdon, 1962). The cytoplasm therefore induces the transplanted nucleus to follow the normal programme of changes in the rate and frequency of DNA synthesis and cell division.

4 Injected native DNA produces similar effects to nuclei. Thus native DNA stimulated DNA synthesis in eggs (Gurdon, Birnstill, and Speight, 1969), but not when injected into oocytes (Gurdon and Speight, 1969; Ford and Woodland, in preparation). This shows that in these cells the replication of DNA and its control does not require nuclear components other than DNA itself.

5 Denatured DNA stimulates DNA synthesis in both eggs (Gurdon et al, 1969) and oocytes (Ford and Woodland, in preparation). It therefore seems to produce a synthetic activity subject to control mechanisms different from those regulating DNA replication in nuclei.

6 When cytoplasm is transferred from a maturing oocyte to a normal, large oocyte it is induced to mature (Smith and Ecker, 1969; Masui and Markert, 1971). Any nuclei injected into the animal pole region of a maturing egg mimic the behaviour of the oocyte nucleus, their nuclear membranes breaking down and their chromosomes condensing and associating with cytasters (Gurdon, 1968). Therefore these processes, which may be compared to the mitosis of somatic cells, are under cytoplasmic control. It is a change in the cytoplasm, not the nucleus, which produces the change in nuclear behaviour as the oocyte matures to form an egg.

7 Once the maturation of the oocyte is complete, the cytoplasm of this non-dividing cell prevents mitosis when injected into cleaving one- or two-cell embryos (Masui and Markert, 1971). There thus seems to be a cytoplasmic factor capable of preventing cell division.

These conclusions indicate that nuclear division and DNA synthesis are under immediate cytoplasmic control-although it may well be that the cytoplasmic control systems are constructed under the nuclear direction in the long term. It therefore seems possible that the abnormalities responsible for the excessive division rates of cancerous cells might be found in the cytoplasm, as well as in the nucleus.

There are grounds for hope that the sort of experiment described above may be helpful in revealing the molecular basis for the control of cell multiplication. Such experiments could be extended to include the injection of the nuclei and other components of malignant cells. They might then be of direct help in localizing the lesion which permits cancerous cells to escape the regulatory mechanisms normally limiting cell division rates. Some experiments of this general nature are described in the rest of this review.

\section{The Analysis of the Developmental Potentiality of} Nuclei

NORMAL CELLS

The most stringent test by which the normality of a nucleus may be assessed is that in which it is asked to programme the complete development of a new organism. This is achieved by removing or destroying the haploid female nucleus of the egg and, by microinjection, replacing it with the nucleus of a somatic cell. This sort of experiment has so far been carefully employed only in the Amphibia. Initially it was shown by King and Briggs (1954) using Rana pipiens, and by Fischberg, Gurdon, and Elsdale (1959) using Xenopus laevis, that the nuclei of early embryonic cells programme normal development with high frequency. Later experiments showed that the nuclei of functionally specialized cells were also able to do this, although with a much lower frequency. For example, nuclei from the absorptive cells of the tadpole intestinal epithelium form adult fertile frogs (Gurdon, 1962; Gurdon and Uehlinger, 1966), and nuclei of cells cultured from the lung, kidney, and skin of adult frogs form fairly normal tadpoles (Laskey and Gurdon, 1970). These experiments show that the nuclei of normal, determined 
cells remain capable of directing the appearance of the normal spectrum of the diverse types of cell characteristic of a vertebrate, each in its normal position. Therefore, no irreversible change in the genetic machinery of the nucleus need accompany cell specialization.

\section{CANCEROUS CELLS}

Our understanding of the cancerous state would be greatly helped by any experiment which showed whether the nuclei of malignant cells are normal, or whether they contain a genetic alteration responsible for the malignancy. One experiment of this sort is that in which the nuclei of malignant cells are transplanted into enucleated eggs to determine their development potentiality. This has been attempted by King, DiBerardino, McKinnell, and their associates, who used a naturally occurring adenocarcinoma of Rana pipiens, first described by Lucké (1934). This is a virus-associated tumour derived from cells in the proximal segment of the nephron, and the animals used are usually obtained from wild populations, in which they occur quite commonly.

The general design of the experiments was to dissociate the tumour into individual cells, and to inject these into manually enucleated, unfertilized eggs of $R$. pipiens. In some of the studies several nuclei were injected, whereas in others single nuclei were transferred (eg, King and DiBerardino, 1965). The first complete study published was that of King and DiBerardino (1965), who found that as many as $4 \%$ of the tumour tissue nuclei produced completely cleaved blastulae in the first nuclear transfer generation (table I). It is important to note that this figure is no lower than that obtained after transferring the nuclei of normal kidney cells. If the blastulae produced by this first transplant operation were used as donors to produce a second transfer generation, as many as $40 \%$ of the recipient eggs formed complete blastulae. If the blastulae were allowed to develop, none of those derived from normal kidney cells proceeded much further, whereas quite high proportions of those from the tumour cells became tadpoles (table I). Although none of these were normal and all died before metamorphosis, they contained most, and perhaps all of the usual complement of cell types, in a conformation that was very near normality. This study thus suggests that some tumour tissue cells contained the full complement of genes necessary for forming the specialized cells of the tadpole. In addition, their nuclei must have been able to respond to the regulatory circuits of the egg cytoplasm in a normal manner. In particular, they must have shown accurate responses to the stimuli responsible for controlling DNA synthesis and nuclear division. This is not a conclusion one might have expected to make of cancerous cells if their regulatory abnormalities were produced by changes at the genetic level.

One doubt these experiments raised concerned the participation of the egg nucleus in the development of the embryos. This is important because the frequency of normal development was low and the efficiency of enucleation may not have been $100 \%$. This doubt can be rigorously eliminated only by using a nuclear marker for identifying the donor nuclei. This has now been done by McKinnell, Deggins, and Labat (1969), who used triploid frogs injected with the carcinogenic virus. Table II shows that in their experiments $5-8 \%$ of the eggs receiving

\begin{tabular}{|c|c|c|}
\hline Source of Cells ${ }^{1}$ & $\begin{array}{l}\text { Number of } \\
\text { Transplants }\end{array}$ & $\begin{array}{l}\text { Percentage of Transplants } \\
\text { Forming Swimming } \\
\text { Tadpoles }\end{array}$ \\
\hline $\begin{array}{l}\text { Disaggregated triploid } \\
\text { adenocarcinoma }\end{array}$ & 143 & $5^{2}$ \\
\hline $\begin{array}{l}\text { Disaggregated diploid } \\
\text { adenocarcinoma }\end{array}$ & 181 & $8^{3}$ \\
\hline
\end{tabular}

Table II Success of nuclear transplant experiments with adenocarcinoma cells bearing nuclear markers (from McKinnell et al, 1969)

${ }^{1}$ In each transfer multiple nuclei were injected.

'Five of the seven tadpoles obtained were shown to be triploid. ${ }^{3}$ All of the embryos were shown to be diploid.

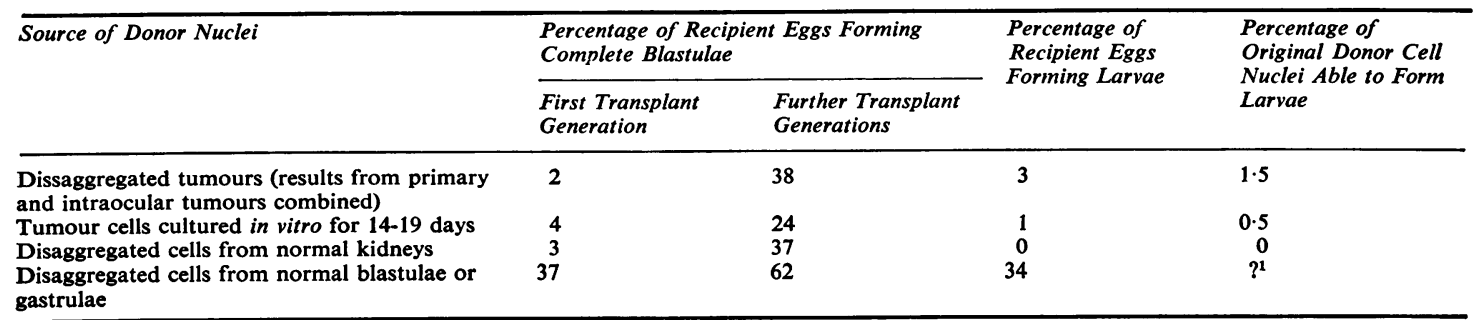

Table I Success of development promoted by adenocarcinoma nuclei (from King and Di Berardino, 1965)

${ }^{1}$ In experiments using Rana this figure would usually be about $60 \%$, but the authors gave insufficient evidence for it to be calculated for this study. 
tumour tissue nuclei developed to the tadpole stage. Since these tadpoles were diploid or triploid as appropriate, it appears that the nuclei of tumour cells were capable of forming the diverse tissues of a tadpole without the assistance of any other nuclear genes.

The most meaningful statistic which one would like to derive from the experiments described above is the proportion of tumour cells containing nuclei able to direct the development of larvae. There are two reasons why it is difficult to do this. First, King and DiBerardino (1965) obtained most of their larvae from the second transplant generation. Since only two first generation donors were used for the serial transfers, it is only possible to conclude that $1 \%$ of the tumour tissue cell nuclei were totipotent. Of course, this is a minimum value, since there is a plethora of trivial technical reasons why nuclear transplant operations using adult somatic cells should fail. However, the fact remains that firm conclusions can be drawn about only $1 \%$ of the cells. Secondly, although McKinnell et al (1969) did not study serial transfer generations, they transferred several nuclei into each egg. It is therefore not certain if they were testing the developmental potentiality of one or of more cells. The proportion of the tumour tissue nuclei which were totipotent might therefore have been lower than the $5-8 \%$ success rates of their experiments.

Even with the provisos mentioned above, it is possible to conclude that the genes of at least $1-5 \%$ of the nuclei of Lucké tumour tissue are nearly, or wholly, normal. The importance of this statement is diminished by the fact that tumours normally contain a mixture of malignant and non-malignant cells. Since the malignancy of each cell was not determined before transfer it is not possible to say whether the $1-5 \%$ of nuclei giving nearly normal transplant-embryo development were derived from cancerous cells or from non-cancerous cells present in the tumour tissue. In order to justify such a statement it would be necessary to clone the putative malignant cells, then test the malignancy of some members of the clone and the developmental potentiality of others. Such a course of study would be no mean undertaking, but it is likely that the results obtained would justify the attempt.

\section{Conclusions}

The studies of amphibian development, briefly reviewed above, may already be seen to be providing insight into the mechanisms by which the reproduction of normal cells is controlled. They also provide an opportunity for studying the lesions of malignant cells in a unique way. The conclusions these studies should permit will be immediately applicable only to certain cells of Amphibia. However, it is likely that processes occurring within the cells of all vertebrates are regulated in a similar fashion, and hence that their cancerous cells will be malignant for similar reasons. There is therefore good reason to believe that studies of Amphibia may shed some light on the nature of the cancerous state in man.

I wish to thank Dr J. B. Gurdon, FRS, and Dr O. R. Reeves for comments on the manuscript.

\section{References}

Deuchar, E. M. (1958). Regional differences in catheptic activity in Xenopus laevis embryos. J. Embryol. exp. Morphol., 6, 223-237.

Fischberg, M., Gurdon, J. B., and Elsdale, T. R. (1959). Nuclear transfer in Amphibia and the problem of the potentialities of the nuclei of differentiating tissues. Exp. Cell Res., Suppl., 6, 161-178.

Graham, C. F., Arms, K., and Gurdon, J. B. (1966). The induction of DNA synthesis by frog egg cytoplasm. Develop. Biol., 14, 349-381.

Graham, C. F., and Morgan, R. W. (1966). Changes in the cell cycle during early amphibian development. Develop. Biol., 14, 439460.

Gurdon, J. B. (1962). The developmental capacity of nuclei taken from intestinal epithelium cells of feeding tadpoles. J. Embryol. exp. Morphol., 10, 622-640.

Gurdon, J. B. (1967). On the origin and persistence of a cytoplasmic state inducing nuclear DNA synthesis in frogs' eggs. Proc. nat. Acad. Sci. (Wash.), 58, 545-552.

Gurdon, J. B. (1968). Changes in somatic cell nuclei inserted into growing and maturing amphibian oocytes. J. Embryol. exp. Morphol., 20, 401-414.

Gurdon, J. B., Birnstiel, M. L., and Speight, V. A. (1969). The replication of purified DNA introduced into living egg cytoplasm. Biochim. biophys. Acta (Amst.), 174, 614-628.

Gurdon, J. B., and Speight, V. A. (1969). The appearance of cytoplasmic DNA polymerase activity during the maturation of amphibian oocytes into eggs. Exp. Cell Res., 55, 253-256.

Gurdon, J. B., and Uehlinger, V. (1966). 'Fertile' intestine nuclei. Nature (Lond.), 210, 1240-1241.

Gurdon, J. B., and Woodland, H. R. (1968). The cytoplasmic control of nuclear activity in animal development. Biol. Rev., 43, 233-267.

Gurdon, J. B., and Woodland, H. R. (1970). On the long-term control of nuclear activity during cell differentiation. Curr. Top. in Develop. Biol., 5, 39-70.

Izawa, M., Allfrey, V. G., and Mirsky, A. E. (1963). Composition of the nucleus and chromosomes in the lampbrush stage of the newt oocyte. Proc. nat. Acad. Sci. (Wash.), 50, 811-817.

King, T. J., and Briggs, R. (1954). Transplantation of living nuclei of late gastrulae into enucleated eggs of Rana pipiens. J. Embryol. exp. Morphol., 2, 73-80.

King, T. J., and DiBerardino, M. A. (1965). Transplantation of nuclei from the frog renal adenocarcinoma. I. Development of tumor nuclear-transplant embryos. Ann. N.Y. Acad. Sci., 126, 115-126.

Laskey, R. A., and Gurdon, J. B. (1970). Genetic content of adult somatic cells tested by nuclear transplantation from cultured cells. Nature (Lond.), 228, 1332-1334.

Lucké, B. (1934). A neoplastic disease of the kidney of the frog, Rana pipiens. Amer. J. Cancer, 20, 352-379.

McKinnell, R. G., Deggins, B. A., and Labat, D. D. (1969). Transplantation of pluripotential nuclei from triploid frog tumors. Science, 165, 394-396.

Masui, Y., and Markert, C. L. (1971). Cytoplasmic control of nuclear behavior during meiotic maturation of frog oocytes. $J$. exp. Zool., 177, 129-145.

Smith, L. D., and Ecker, R. E. (1969). Role of the oocyte nucleus in physiological maturation in Rana pipiens. Develop. Biol., 19, 281-309. 
Smith, L. D., and Ecker, R. E. (1970). Regulatory processes in the maturation and early cleavage of amphibian eggs. Curr. Top. in Develop. Biol., 5, 1-38.

Woodland, H. R., Ford, C. C., Gurdon, J. B., and Lane, C. D. (1972). Some characteristics of gene expression as revealed by a living assay system. In Molecular Genetics and Developmental
Biology, edited by M. Sussman, pp. 393-423. Prentice-Hall, New Jersey.

Woodland, H. R., and Gurdon, J. B. (1968). The relative rates of synthesis of DNA, sRNA and rRNA in the endodermal region and other parts of Xenopus laevis embryos. J. Embryol. exp. Morphol., 19, 363-385.

.

王

